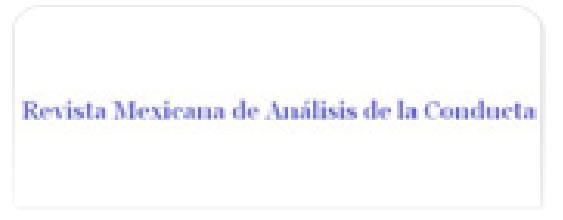

Revista Mexicana de Análisis de la Conducta ISSN: 0185-4534

editora@rmac-mx.org

Sociedad Mexicana de Análisis de la Conducta México

Fajardo-Vargas, Violeta; Hernández-Guzmán, Laura

Tratamiento cognitivo-conductual de la conducta agresiva infantil

Revista Mexicana de Análisis de la Conducta, vol. 34, núm. 2, diciembre, 2008, pp. 371-389

Sociedad Mexicana de Análisis de la Conducta

Guadalajara, México

Disponible en: http://www.redalyc.org/articulo.oa?id=59311115013

Cómo citar el artículo

- Número completo

- Más información del artículo

- Página de la revista en redalyc.org

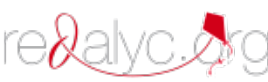

Sistema de Información Científica

Red de Revistas Científicas de América Latina, el Caribe, España y Portugal Proyecto académico sin fines de lucro, desarrollado bajo la iniciativa de acceso abierto 


\title{
TRATAMIENTO COGNITIVO-CONDUCTUAL DE LA CONDUCTA AGRESIVA INFANTIL
}

\author{
COGNITIVE-BEHAVIORAL TREATMENT OF AGGRESSIVE \\ BEHAVIOR IN CHILDREN
}

VIOLETA FAJARDO-VARGAS Y LAURA HERNÁNDEZ-GUZMÁN' ${ }^{1}$ UNIVERSIDAD NACIONAL AUTÓNOMA DE MÉXICO

\begin{abstract}
RESUMEN
El presente estudio investigó si la combinación de tratamiento cognitivo-conductual dirigido a los niños, y el entrenamiento de sus padres sobre cómo manejar el comportamiento infantil, es más eficaz que el solo tratamiento a los niños, tanto desde el punto de vista del análisis estadístico del cambio, como desde el de la relevancia clínica. También, exploró si los efectos del tratamiento se reflejan en el funcionamiento social infantil y en el estrés de sus padres. Si bien, desde el punto de vista estadístico, ambas condiciones por igual mostraron mejoría tanto de la agresividad infantil como de los problemas sociales observados por sus padres, el análisis de la relevancia clínica reveló la superioridad del grupo combinado. El estrés de los padres no cambió a partir del tratamiento. Palabras clave: terapia conductual, eficacia, parental, intervención
\end{abstract}

\begin{abstract}
The aim of the present study was to investigate the efficacy of the combination of parental training in behavioral management techniques in children with aggressive behavior along with cognitive behavioral treatment applied to chil-

1. Correspondencia: Dra. Laura Hernández-Guzmán, Av. Universidad 3004, Edificio D, Laboratorio I, Col. Copilco-Universidad, C.P. 04510, Distrito Federal, México, Iher@unam.mx

Recibido: 17 de Noviembre, 2008. Revisado: 28 de Diciembre, 2008. Aceptado: 30 de Diciembre, 2008
\end{abstract}


dren, as compared to the sole treatment of children. The effects of both type of treatments (combined and treatment alone) at posttest were also analyzed from a traditional statistical approach, as well as from the point of view of its clinical significance. Also, the effect of treatment was explored in children's social functioning and parental stress. According to statistical analyses, both conditions decreased children's aggression and social problems as perceived by their parents; however, only combined treatment was clinically significant. Parental stress did not change after treatment in either group. Key words: behavior therapy, efficacy, parents, intervention

La agresividad infantil se ha definido en términos de la conducta manifiesta del niño que implica destrucción, daño físico a otros, a él mismo, o a propiedades. Entraña agresión física, amenazas verbales de agresión, arrebatos explosivos de destrucción de propiedad y auto-daño en el contexto de frustración o estimulación aversiva.

Si no se trata oportunamente, por ser un problema con inicio en la infancia, no sólo tiende a persistir en la edad adulta, sino que se exacerba con el paso del tiempo. Es precursora la conducta agresiva, en gran medida, de comportamientos antisociales a corto y mediano plazos, tales como la criminalidad y la delincuencia (Kazdin \& Buela-Casal, 1994; Widom, Schuck, \& White, 2006), el abuso de sustancias (Spatz-Widom \& Hiller-Sturmhofel, 2001), y las relaciones sexuales tempranas (Caspi \& Moffitt, 1995; Fergusson, Horwood \& Ridder, 2005; Rhule, 2005). Además, repercute sobre otros desenlaces como el fracaso escolar y el rechazo de los compañeros (Patterson, DeBaryshe \& Ramsey, 1989). Las repercusiones de la conducta agresiva se asocian con la pérdida de productividad y la necesidad de intervenciones continuas a corto y largo plazos (Leve \& Chamberlain, 2005; Rhule, 2005). Consecuencias que, de acuerdo con Kazdin y Buela-Casal (1994), tienden a agotar los recursos de la comunidad, como son los servicios de salud, la educación y los sistemas de justicia.

Identificada como un ingrediente común a los problemas de conducta y las conductas antisociales, la conducta agresiva representa uno de los problemas más frecuentes de salud mental en los niños (Smeekens, RiksenWalraven \& van Bakel, 2007), aproximadamente oscila entre un $30 \%$ y un $50 \%$ de todos los problemas clínicos que se refieren a consulta (Kazdin, 1987, 1996). En México, si bien no se cuenta, al parecer, con datos concretos sobre su prevalencia en la población infantil, se han documentado algunas manifestaciones asociadas con ésta, como son la inquietud 19\%, la irritabilidad $17 \%$, el nerviosismo $16 \%$, el déficit de atención $14 \%$, la desobediencia $13 \%$, la explosividad $11 \%$ y la conducta dependiente $9 \%$ (Caraveo-Anduaga, Colmenares-Bermúdez \& Martínez-Vélez, 2002).

La psicopatología, al igual que la conducta normal, se desarrolla a partir de las experiencias vitales del individuo. Es la transacción con el ambiente 
circundante, la que explica con mayor fuerza el desarrollo de la conducta, ya sea ésta adaptada o disfuncional (Hernández-Guzmán, 1999; Novak, 1998). En el caso de la agresividad, existen factores, tales como el temperamento del niño y su inhabilidad para regular sus emociones y conducta que lo predisponen a transacciones defectuosas con su ambiente circundante. Por ser el sistema familiar el contexto más relevante durante la niñez, se ha sugerido que es el que más factores podría aportar a su aparición y mantenimiento. La conducta agresiva aparece y se estabiliza a partir de la transacción continua del niño con su ambiente inmediato, específicamente de la forma en que sus padres o cuidadores principales ejercen la crianza (Chorpita \& Barlow, 1998; Wagner, 1997). Es alta la probabilidad de que un niño impulsivo, con un control emocional precario, se exponga a experiencias de castigo y coerción por parte de sus padres (Smeekens, et al. 2007; Rubin, Burgess, Dwyer \& Hastings, 2003). Cuando los padres recurren al castigo físico o a otras formas de coerción psicológica, como recurso para disciplinar a sus hijos, lejos de resolver el problema, lo exacerban (Castell, Frías, Corral \& Sotomayor, 2000; Criss, Pettit, Bates, Dodge \& Lapp, 2002; Landsford et al., 2005; Levy, 1999; Muris, Bögels, Meesters, van der Kamp \& van Oosten, 1996; Nelson, Hart, Yang, Olsen \& Jin 2006; Simons, Lin \& Gordon, 1998; Smeekens, et al., 2007; Straus, Hamby, Finkelhor, Moore \& Runyan, 1998; Su, 1975; Vitaro, Barker, Boivin, Brendgen \& Tremblay, 2006).

Si bien el castigo se ha señalado como una variable que exacerba la agresividad infantil, otros estudios sugieren que además del castigo, un factor de riesgo importante, responsable del desarrollo de la agresividad en los hijos, es el manejo ineficaz de su comportamiento, caracterizado por esfuerzos fallidos, inconsistentes y erráticos para disciplinarlos (Dumas, 1992; Haapasolo \& Pokela, 1999; Kingston \& Prior, 1995; Jaffee, Caspi, Moffitt \& Taylor, 2004; Moszczynska, 1979; Sim \& Ong, 2005; Stormont-Spurgin \& Zentall, 1996; Su, 1975).

El hecho de que los padres contribuyan de diversas formas a la agresividad de sus hijos ha sugerido la necesidad de diseñar intervenciones, tanto preventivas como terapéuticas, dirigidas a los padres o cuidadores principales mediante la modificación de sus prácticas disciplinarias. Por lo tanto, la prevención y el tratamiento de la agresividad infantil se han beneficiado también de la investigación sobre técnicas conductuales diseñadas para alterar las transacciones padres-hijo en el hogar.

Los datos de investigación sugieren que el entrenamiento de los padres no es una estrategia suficiente por sí sola para modificar la conducta agresiva infantil. Por otro lado, los resultados de las diversas investigaciones en este campo tampoco apoyan suficientemente el tratar sólo a los niños. Coinciden, así, los diversos autores en que la estrategia más exitosa es la combinación del tratamiento a los niños en solución de problemas y formación de habilida- 
des, por un lado, y el manejo conductual del niño mediante el entrenamiento de sus padres, por el otro. Webster-Stratton y Hammond (1997) hallaron que la intervención conductual por parte de los padres combinada con el tratamiento en solución de problemas a los niños es más eficaz que el tratamiento dirigido sólo a los niños y el entrenamiento sólo a los padres. Aunque cada uno de éstos produce cambios importantes, la combinación de ambos es más eficaz. Otros estudios han corroborado este hallazgo y añaden que el tratamiento dirigido a los niños es más eficaz que el tratamiento proporcionado sólo a los padres, pero que la combinación del tratamiento dirigido a los niños y a sus padres es aún más eficaz (Brestan \& Eyberg, 1998; Kazdin, 1996; Kazdin, Siegel \& Bass, 1992; Tynan, Chew \& Algermissen, 2004).

A pesar de que las 3 estrategias de tratamiento tienden a reducir la conducta agresiva y a aumentar la competencia social, el tratamiento combinado muestra efectos mucho más claros y duraderos, de acuerdo con Kazdin, et al. (1992). También se observa coincidencia de una investigación a otra en que las estrategias cognitivo-conductuales son las más eficaces para reducir la agresividad infantil. Concretamente, las técnicas cognitivo-conductuales más eficaces, de acuerdo con la mayoría de los estudios en población anglosajona, son la solución de problemas en el caso de los niños agresivos (Kazdin, Esveldt-Dawson, French \& Unis, 1987), el entrenar a los padres a aplicar los principios de la conducta al disciplinar a sus hijos (Forehand \& Atkenson, 1977; Marshall \& Mirenda, 2002; Miller, Kuschel \& Hahlweg, 2002; Patterson, Chamberlain \& Reid, 1982; Peed, Roberts \& Forehand, 1977), y la combinación de ambas (Froelich, Doepfner, Berner \& Lehmkuhl, 2002; Hemphill \& Littlefield, 2001). Cabe señalar que no basta con demostrar mejoría a partir del tratamiento. Para que un tratamiento se considere validado empíricamente y recomendable para diversas poblaciones, un equipo de investigadores, distinto al que hubiese demostrado originalmente su eficacia, debe encontrar resultados similares (Hamilton \& Dobson, 2001). Es una asignatura pendiente indagar si esos resultados se repiten en niños mexicanos.

Finalmente, dentro de esta línea de investigación, la eficacia de las intervenciones psicológicas se ha estudiado solamente en términos de la reducción de las conductas disfuncionales. Es por ello que resulta importante evaluar también la relevancia clínica del tratamiento por medio del análisis del cambio en cada individuo. En la medida en la que más individuos expuestos al tratamiento califiquen dentro de la normalidad, es decir dentro de los puntos de corte establecidos, mayor relevancia clínica (Nietzel \& Trull, 1988). Asimismo, Kazdin (2001) ha propuesto que se considerará más efectivo un tratamiento, en la medida en la que su efecto se refleje en el funcionamiento de los niños en su contexto social, y en el estrés de los padres. Si la mera condición de ser padre impone demandas, el contender con un hijo agresivo implica, para algunos padres, una fuente constante de estrés, lo que alte- 
ra no sólo su funcionamiento biológico, sino también, el psicológico (Miller, 1989). El problema se complica aún más, pues el estrés que experimentan los padres suele afectar la forma en que disciplinan a sus hijos. Se sabe que, aún ante la transgresión más leve de los hijos, un padre o una madre estresada(o) tiende a mostrarse más irritable, a centrar su atención en la conducta desviada, y a iniciar y exacerbar intercambios agresivos con el hijo (Anthony et al., 2005; Huth-Bocks, \& Hughes, 2008; Pinderhughes, Dodge, Bates, Pettit \& Zelli, 2000).

El presente estudio, por lo tanto, pretende investigar si el tratamiento combinado, dirigido, tanto a los niños, como a sus padres, es más eficaz que el solo tratamiento a los niños, tanto desde el análisis estadístico tradicional, como desde la perspectiva de la relevancia clínica. También, resulta relevante explorar si los efectos del tratamiento se reflejan en el funcionamiento infantil dentro de su contexto social y en el estrés de sus padres. De acuerdo con los resultados anteriormente revisados, se espera que (a) el tratamiento de solución de problemas dirigido sólo a los niños reduzca eficazmente su conducta agresiva, (b) la combinación del tratamiento a los niños con el entrenamiento proporcionado a los padres sobre cómo manejar el comportamiento infantil reduzca la agresividad en mayor proporción que el tratamiento aplicado sólo a los niños, y (c) a la vez que disminuya la agresividad de los niños, mejoren sus problemas sociales y disminuya el estrés manifestado por sus padres.

\section{MÉTODO}

\section{Participantes}

Inicialmente, los padres de 1935 niños de diversos colegios públicos de diferentes zonas marginadas de la Ciudad de México respondieron a la Lista de Conductas de Achenbach (CBCL 6-18, por sus siglas en inglés) (Achenbach \& Rescorla, 2001). Se seleccionó intencionalmente a 100 niños, entre 6 y 12 años, que puntuaron dentro del área clínica de la escala de agresividad del $\mathrm{CBCL}$. Se ofreció la oportunidad a sus padres de participar en el estudio mediante la firma de una carta de consentimiento. Sólo 42 consintieron que sus hijos participaran en el estudio, 13 niñas y 29 varones. Se les asignó al azar a uno de 2 grupos, un grupo conformado por 22 niños en el que participaron sólo ellos y uno combinado, de 20 niños, en el que participaron también sus padres.

Instrumentos y materiales

La Lista de Conductas de Achenbach (Children Behavior Checklist, CBCL/618; Achenbach \& Rescorla, 2001) que contestan los padres es un instrumento que mide una gama amplia de conductas internalizadas y externalizadas 
de los niños, compuesto por 118 ítems que contienen preguntas para los padres (alfa $=0.96)$ (Hernández-Guzmán, 2003). Sus ítems se agrupan en 9 factores (ansioso/deprimido, aislado/deprimido, quejas somáticas, problemas sociales, problemas de pensamiento, problemas de atención, conducta de violar reglas, conducta agresiva y otros problemas). Se califica $0=$ nunca, $1=$ a veces y $2=$ siempre. Para los análisis del presente estudio se utilizó solamente la calificación obtenida en las escalas de agresividad (alfa $=.89$ ) y problemas sociales $($ alfa $=0.71$ ). La escala de agresividad está conformada por 18 ítems, como por ejemplo, discute por todo, es cruel, molesta a otros, es malo(a) con los demás, y tiene un punto de corte de 11 puntos. Por su parte, la escala de problemas sociales consta de 11 reactivos que miden problemas sociales, como por ejemplo, se queja de estar solo(a), no se lleva bien con otros niños.

\section{Estrés Parental}

Escala de Estrés Parental (Hernández-Guzmán \& Benjet, 2003) contiene 32 ítems tipo Likert con 3 opciones de respuesta, "falso", "a veces cierto", "cierto" que contestan los padres. Esta escala presenta un alfa de 0.83 . Evalúa el estrés relacionado con la condición de ser padre/madre, y estrés debido a eventos vitales. La escala de Estrés Parental se compone de 8 sub-escalas: disciplina o manejo ineficaz, desacuerdo con la pareja, apoyo de la familia, desacuerdo con la familia, goce de la paternidad, evasión de la paternidad, rechazo de la paternidad y educación negativa. A mayor calificación más estrés parental, la calificación más alta posible es 64 .

\section{Diseño experimental}

Se empleó un diseño experimental de 2 grupos asignados aleatoriamente a 2 condiciones y 2 momentos de evaluación pre- y post-tratamiento. Cada grupo se expuso a una de dos condiciones: (a) el tratamiento de la agresividad dirigido a los niños, (b) el entrenamiento a los padres para manejar los problemas de agresividad de sus hijos, a la vez que éstos se sometían al tratamiento de su agresividad.

\section{PROCEDIMIENTO}

\section{Evaluación pre-tratamiento}

Los padres de los 42 niños agresivos cumplimentaron, además de la Lista de Conductas de Achenbach (CBCL/6-18), la Escala de Estrés Parental. 
Tratamiento

Se asignó aleatoriamente a los niños a uno de los 2 grupos, uno formado por niños cuyos padres se entrenaron en el manejo conductual de la agresividad, y otro por niños que sólo recibieron la intervención. Los niños de ambos grupos se expusieron al mismo tratamiento. Se programaron las sesiones con los padres y con los niños, dos veces por semana.

En el curso de 5 semanas, con base en un manual de tratamiento construido expresamente para este estudio, 2 terapeutas previamente entrenados aplicaron la intervención en forma grupal a los 42 niños asignados a las 2 condiciones de tratamiento. Se formaron 7 equipos de 6 niños cada uno que se sometieron a 10 sesiones de tratamiento basado en técnicas cognitivoconductuales, con duración de 60 minutos cada una. Todos los niños recibieron, por lo tanto, el mismo tratamiento. El tratamiento incluyó, en primer término, la psicoeducación, mediante la cual el niño aprende a describir qué es la agresividad y define operacionalmente su propia agresividad. En segundo lugar, se aplicó la técnica de solución de problemas (D'Zurilla \& Nezu, 1999), mediante la cual el niño identifica soluciones a problemas específicos que enfrenta en la vida cotidiana. Un problema se definió como cualquier suceso o situación que actúe como una amenaza o discrepancia entre lo que uno quiere y la realidad. Una solución en este contexto se refiere a cualquier respuesta de afrontamiento al problema. Las soluciones efectivas, sin embargo, son aquéllas en donde la respuesta de afrontamiento no sólo resuelve el problema, sino que maximiza las consecuencias positivas y minimiza las negativas. Finalmente, se entrenó al niño en habilidades sociales, cuyo objetivo es formar conductas prosociales mediante el modelamiento, las aproximaciones sucesivas y la imaginación guiada. También se enseñó al niño a relajarse.

Por otra parte, los padres de los 20 niños asignados al grupo que incluyó entrenamiento a los padres se dividieron en equipos de 4 a 10 personas. Cada equipo recibió entrenamiento durante 10 sesiones de una hora por parte de 2 terapeutas previamente entrenados. Podían participar ambos padres o sólo uno. El entrenamiento de los padres intenta modificar las prácticas de crianza, incluye la psicoeducación, que les permite identificar la agresividad, definir operacionalmente la conducta agresiva y el comportamiento prosocial de sus hijos. Se enseñó también a los padres a aplicar el reforzamiento contingente al comportamiento prosocial del niño y a establecer límites.

\section{Evaluación post-tratamiento}

Inmediatamente después de la última sesión, los padres contestaron nuevamente el CBCL/6-18 y la Escala de Estrés Parental. 


\section{RESULTADOS}

El análisis grupal de los datos sobre Agresividad, Problemas Sociales y Estrés de los Padres se efectuó por separado mediante ANOVA de dos factores con medidas repetidas en un factor con el propósito de observar el efecto de la intervención sobre cada una de estas variables dependientes.

\begin{tabular}{|c|c|c|c|c|}
\hline & \multicolumn{2}{|c|}{ Agresividad } & & \\
\hline & & Pre & \multicolumn{2}{|c|}{ Post } \\
\hline & Media & $D E$ & Media & $D E$ \\
\hline Sólo niños & 16.01 & 4.765 & 10.59 & 5.781 \\
\hline Combinado & 17.88 & 4.123 & 9.34 & 3.669 \\
\hline Total & 16.90 & 4.516 & 9.99 & 4.874 \\
\hline
\end{tabular}

Tabla 1. Medias y desviaciones estándar de las puntuaciones obtenidas por los niños en el factor agresividad del CBCL/6-18 en las mediciones pre- $y$ post-tratamiento.

En la tabla 1 aparecen las medias y desviaciones estándar para las condiciones de tratamiento dirigido sólo a los niños y el combinado en las evaluaciones de pre tratamiento y post tratamiento.

En el factor agresividad, la diferencia antes $(M=16.90)$ y después del tratamiento $(M=9.99)$ resultó significativa, con una $F(1,40)=64.149, p<$ 0.000 , para ambas condiciones.

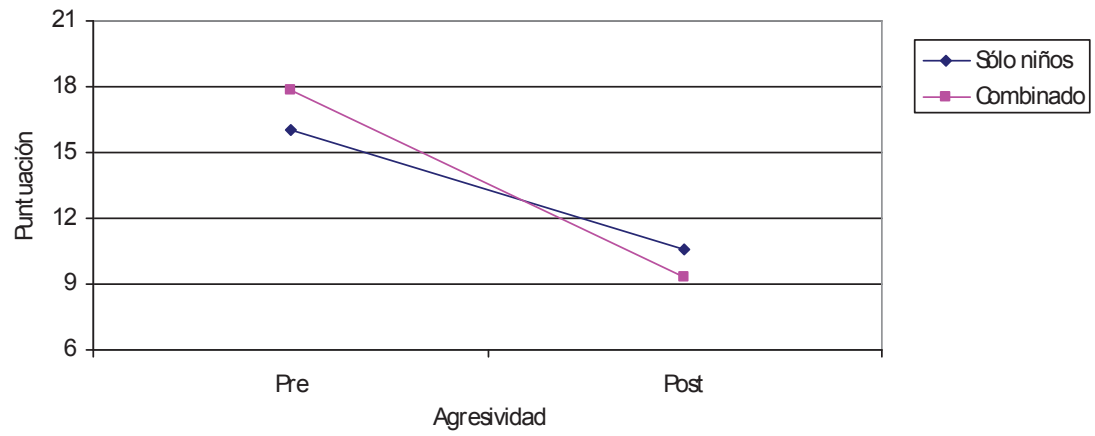

Figura 1. Comparación de los efectos del tratamiento sobre la conducta agresiva. 
La figura 1 muestra la interacción entre las condiciones de tratamiento con medidas repetidas en el factor agresividad (antes y después) con una $\mathrm{F}$ (1, $40)=3.194, p<0.081$. No se encontraron diferencias significativas en la interacción entre agresividad (antes y después) y condición de tratamiento. Es decir, se observó una disminución de la conducta agresiva del pre tratamiento y post tratamiento independientemente de la condición de la que se tratara.

\begin{tabular}{|c|c|c|c|c|c|}
\hline \multicolumn{6}{|c|}{ Condición de Tratamiento } \\
\hline \multicolumn{3}{|c|}{ Solo niños } & \multicolumn{3}{|c|}{ Combinado } \\
\hline Identificación & Pre & Post & Identificación & Pre & Post \\
\hline J.R. & 12 & 12 & A.I. & 15 & 10 \\
\hline D.A. & 13 & 17 & A.I.H. & 17 & 5 \\
\hline A.L. & 12 & 6 & C.A. & 13 & 12 \\
\hline A.G. & 18 & 15 & C.R. & 16 & 12 \\
\hline D.C. & 14 & 5 & D.E. & 13 & 7 \\
\hline J.B. & 15 & 9 & P.S. & 16 & 10 \\
\hline V.G. & 18 & 10 & M.E. & 12 & 4 \\
\hline I.K. & 14 & 10 & D.A. & 13 & 5 \\
\hline A.S. & 13 & 11 & D.V. & 21 & 13 \\
\hline D.R. & 30 & 18 & M.A. & 18 & 14 \\
\hline D.L. & 17 & 9 & J.A. & 17 & 5 \\
\hline A.M. & 12 & 3 & J.L. & 23 & 10 \\
\hline J.S. & 16 & 7 & O.A. & 19 & 5 \\
\hline J.A. & 29 & 4 & A.C. & 22 & 10 \\
\hline P.F. & 14 & 3 & I.B. & 19 & 7 \\
\hline V.V. & 16 & 19 & E.R. & 29 & 16 \\
\hline J.C. & 17 & 21 & U.U. & 19 & 9 \\
\hline L.A. & 15 & 18 & S.O. & 21 & 14 \\
\hline P.E. & 14 & 2 & P.O. & 20 & 6 \\
\hline B.T. & 16 & 13 & D.C. & 15 & 13 \\
\hline O.D. & 15 & 15 & & & \\
\hline G.A. & 12 & 6 & & & \\
\hline
\end{tabular}

Nota. El punto de corte para el factor agresividad del CBCL 6/18 es 11

Tabla 2. Puntuaciones obtenidas por cada niño de ambos grupos en el factor agresividad del $\mathrm{CBCL/6-18}$

Respecto a la relevancia clínica del tratamiento de la agresividad, la tabla 2 presenta las calificaciones individuales en el factor agresividad del CBCL/6- 
18 en la condición de tratamiento sólo a los niños y el combinado (tratamiento a los niños y a sus padres), antes y después de la intervención, respecto al punto de corte que es de 11 puntos. Del grupo de 22 niños que participaron sin sus padres, 13 niños (59\%) puntuaron por abajo o en el punto de corte después del tratamiento. Otros 3 niños (13.64\%) mostraron disminuciones, pero no alcanzaron el punto de corte. Dos niños más $(9.09 \%)$ no observaron cambios y $4(18.18 \%)$ empeoraron después del tratamiento. Por otra parte, 13 de los 20 niños que formaron parte del grupo combinado mostraron calificaciones abajo del punto de corte después del tratamiento, $65 \%$. El resto, 7 niños (35\%), mostraron disminuciones que no alcanzaron el punto de corte.

Problemas sociales

En la tabla 3 se presentan las medias y desviaciones estándar para las condiciones de tratamiento dirigido sólo a los niños y el combinado, antes y después del tratamiento.

\begin{tabular}{|c|c|c|c|c|}
\hline \multicolumn{5}{|c|}{ Problemas sociales } \\
\hline & \multicolumn{2}{|c|}{ Pre } & \multicolumn{2}{|c|}{ Post } \\
\hline & Media & $D E$ & Media & $D E$ \\
\hline Solo niños & 5.90 & 3.811 & 4.50 & 2.874 \\
\hline Combinado & 7.11 & 3.510 & 4.12 & 2.739 \\
\hline Total & 6.47 & 3.677 & 4.32 & 2.783 \\
\hline
\end{tabular}

Tabla 3. Medias y desviaciones estándar de las puntuaciones obtenidas en el factor problemas sociales del CBCL/6-18 en las mediciones pre y post.

En el factor problemas sociales (antes y después), el análisis arrojó una $\mathrm{F}$ (1, $40)=14.576, p<.000$. En ambas condiciones, la diferencia entre la media antes $(M=6.47)$ y la media después del tratamiento $(M=4.32)$ fue significativa.

El análisis de la interacción entre problemas sociales y condición de tratamiento con medidas repetidas (antes y después) no arrojó diferencias significativas, $F(1,40)=1.904, p<.175$ (véase figura 2 ).

En la figura 2 se observa una disminución en el puntaje de problemas sociales en las dos condiciones con respecto al pre tratamiento y post tratamiento. 


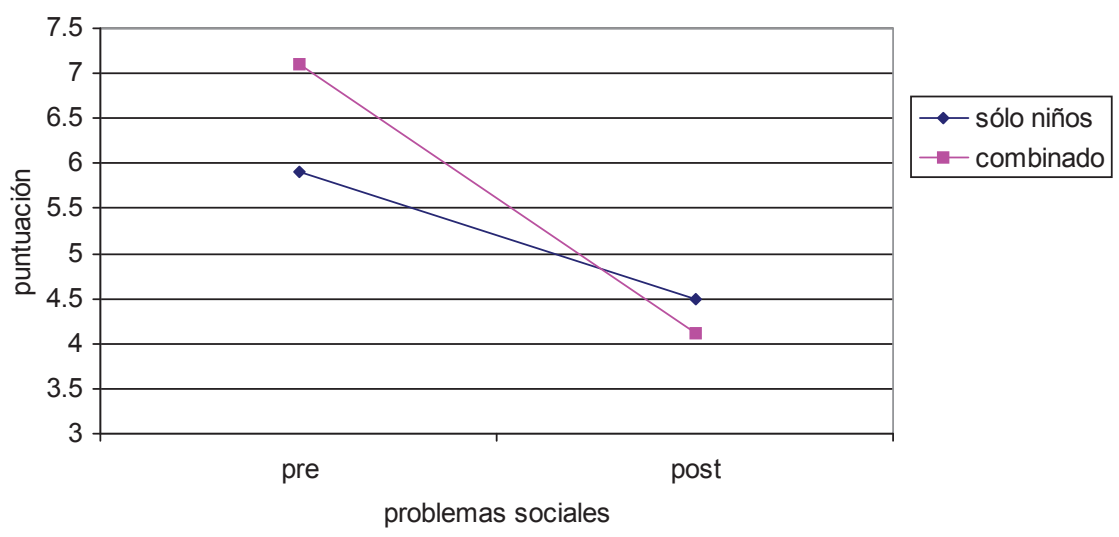

Figura 2. Comparación de los efectos del tratamiento sobre los problemas sociales

\section{Estrés Parental}

En la tabla 4 se presentan las medias y desviaciones estándar para las condiciones de tratamiento dirigido sólo a los niños y el combinado antes y después del tratamiento.

\begin{tabular}{|c|c|c|c|c|}
\hline & \multicolumn{2}{|c|}{ Estrés Parental } & & \\
\hline & \multicolumn{2}{|c|}{ Pre } & \multicolumn{2}{|c|}{ Post } \\
\hline & Media & $D E$ & Media & $D E$ \\
\hline Sólo niños & 15.36 & 5.386 & 17.37 & 7.832 \\
\hline Combinado & 17.10 & 6.719 & 15.13 & 6.772 \\
\hline Total & 16.19 & 6.046 & 16.30 & 7.345 \\
\hline
\end{tabular}

Tabla 4. Medias y desviaciones estándar de las puntuaciones obtenidas en el instrumento de evaluación del estrés parental en las mediciones pre y post.

En el factor estrés parental (antes y después), el análisis arrojó una $F(1,40)$ $=.000, p<.987$. Es decir, no se observó una diferencia significativa entre la media antes $(M=16.19)$ y la media después del tratamiento $(M=16.30)$, en ninguna de ambas condiciones. 
El análisis de la interacción entre el estrés parental versus condición de tratamiento con medidas repetidas (antes y después) arrojó una $F(1,40)=$ $3.838, p<0.057$. La diferencia no fue significativa en la interacción pre y post tratamiento versus condición (figura 3 ).

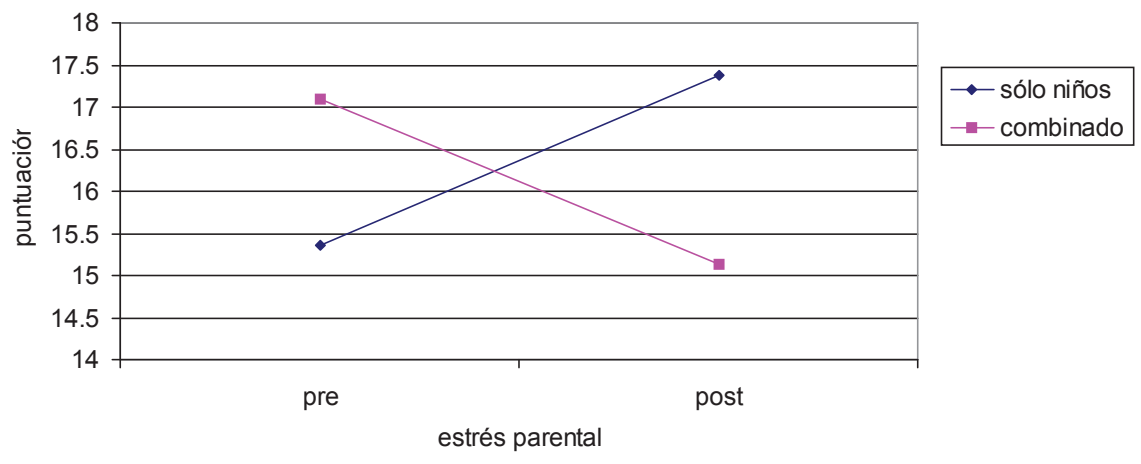

Figura 3. Comparación de los efectos del tratamiento sobre el estrés parental.

En la figura 3 se observa que en tanto que el estrés parental muestra una tendencia a aumentar después del tratamiento para el grupo de padres pertenecientes a la condición de tratamiento dirigido sólo a los niños, tiende a disminuir para la condición de tratamiento combinado. Sin embargo, el cambio no es significativo.

\section{DISCUSION}

El primer propósito del presente estudio fue averiguar si el entrenamiento en el manejo de la agresividad infantil, proporcionado a los padres en combinación con el tratamiento de sus hijos, resultaba mejor que la intervención dirigida sólo a los niños. Se analizó, primero, en cuanto a las diferencias estadísticas entre la evaluación antes y aquélla después del tratamiento. Posteriormente, se exploró la relevancia clínica del tratamiento mediante la inspección de los datos individuales.

Contrario a lo esperado, el grupo combinado, en el que además del tratamiento cognitivo-conductual de los niños se incluyó a sus padres, no resultó superior al grupo en el que participaron solamente los niños. En la escala de 
agresividad del CBCL se observó mejoría en los dos grupos de tratamiento en la evaluación justo después del tratamiento. Este resultado no coincide con los hallazgos de estudios anteriores realizados en otros países que favorecen decisivamente a la intervención combinada (Brestan \& Eyberg, 1998; Kazdin, 1996; Kazdin, et al., 1992; Tynan, et al. 2004; Webster-Stratton \& Hammond, 1997).

El que el entrenamiento a los padres no se reflejara en un efecto diferencial, respecto a la intervención dirigida sólo a los niños, pudo deberse en parte a que, por ser la meta del tratamiento la adquisición de conductas por parte de los padres, como emplear refuerzo positivo e implementar límites al comportamiento de sus hijos, éstas no se reflejaran aún en los resultados obtenidos justo después de terminado el entrenamiento. De otros estudios, se ha documentado que una conducta recién aprendida tarda en establecerse y caer bajo el control de sus consecuencias (Hernández-Guzmán, González \& López, 2002). Se ha observado una mejoría gradual conforme transcurre el tiempo después del tratamiento (Ollendick \& King, 1998). Para poder evaluar este posible efecto, hubiese sido necesario contar con datos de seguimiento, por lo menos 6 meses después del tratamiento. Esto se vio impedido por problemas laborales generalizados en las escuelas del país, y la subsecuente suspensión de labores, cuando correspondía recoger los datos del seguimiento. También, el ausentismo y desinterés observado en los padres que participaron en el entrenamiento podrían explicar el que no se observara una diferencia clara entre ambos grupos. Los padres del presente estudio, pertenecientes a grupos marginados, referían constantemente problemas de tiempo, por la necesidad de trabajar horas adicionales a las que establece la ley o por desempeñar varios trabajos.

De acuerdo con los análisis estadísticos, se apreció mejoría para los dos grupos. Sin embargo, las pruebas estadísticas no necesariamente permiten explorar el progreso o retroceso de cada individuo. El presente estudio, por lo tanto, analizó también la relevancia clínica de ambas modalidades de tratamiento. Varios autores han coincidido en que la relevancia clínica se documenta cuando la persona, una vez sometida al tratamiento, funciona dentro de los niveles de normalidad (Nietzel \& Trull, 1988). Una de las aportaciones del presente estudio fue explorar si hubo cambios clínicamente relevantes para los niños expuestos al tratamiento con o sin sus padres. El análisis individual reveló un panorama distinto al mostrado por el análisis estadístico. Todos los niños del grupo, cuyos padres recibieron entrenamiento, observaron mejoría después del tratamiento, aunque sólo 13 alcanzaron el punto de corte. Sin embargo, el grupo en el que participaron los niños sin sus padres mostró resultados mixtos, casi el mismo porcentaje de niños alcanzó el punto de corte, pero varios niños empeoraron y otros no mostraron cambio alguno. Este resultado que incorpora la perspectiva individual sugiere la superioridad 
del grupo, cuyos padres recibieron entrenamiento sobre el grupo en el que participaron solamente los niños.

Este dato añade validez a los resultados, pues sugiere la relevancia clínica del tratamiento combinado. La mayoría de los niños expuestos al tratamiento combinado cumplieron el criterio de mejoría hasta alcanzar el nivel de normalidad. La relevancia clínica conlleva precisamente ese criterio de funcionamiento normal. Además, los demás niños de este grupo también mejoraron, algunos de ellos disminuyeron drásticamente sus puntuaciones de agresividad, aunque no alcanzaran el punto de corte.

Otro propósito fue indagar si el efecto observado en la agresividad infantil se extendía al funcionamiento social de los niños y al estrés de sus padres. No sólo se observó una mejoría en la evaluación después del tratamiento, en la agresividad, sino que el cambio se reprodujo también en las calificaciones de problemas sociales. Tanto en el factor agresividad como en el factor problemas sociales del CBCL, los niños de los dos grupos mostraron, según sus padres, mejoría en la evaluación posterior al tratamiento. En suma, se observó coincidencia en el efecto observado para ambas condiciones, tanto en la agresividad como en los problemas sociales de los niños. El efecto se repitió, pues, en una segunda variable, problemas sociales, lo que añade validez al resultado. No obstante, esta conclusión debe tomarse con cautela. En primer término, los padres son quienes califican el comportamiento de los niños, tanto la agresividad como los problemas sociales. El empleo del informe de los padres no permite verificar directamente la reducción de las respuestas de agresividad o problemas sociales. Estas medidas basadas en informantes difícilmente pueden descartar la deseabilidad social como explicación de los resultados. Quienes informan tienden a responder de acuerdo con lo que se espera de ellos. Además, estos instrumentos no proporcionan información acerca de las habilidades y la calidad del desempeño de los niños al relacionarse con los demás. La observación directa de los niños en su ambiente social podría ayudar a resolver el problema de la deseabilidad social, por lo que se sugiere incluirla en estudios a futuro.

Respecto al efecto del tratamiento de la agresividad infantil sobre el estrés de los padres, no hubo diferencia significativa de una condición a la otra. Si como han sugerido otros estudios, que el estrés de los padres tiende a recrudecer sus prácticas disciplinarias, aún ante transgresiones menores de los hijos (Anthony et al., 2005; Huth-Bocks \& Hughes, 2008; Pinderhughes et al. 2000), será importante investigar en estudios futuros, si en el caso de los niños que reciben el tratamiento sin sus padres, la agresividad aumenta de nuevo con el paso del tiempo. Como ya se comentó, se echa de menos en el presente estudio los datos de seguimiento que hubiesen arrojado luz también sobre este asunto. 
Además de las ya señaladas, este trabajo presenta limitaciones que resulta importante subrayar. Hubiese sido deseable contar con un grupo en la lista de espera. Por razones éticas, se tomó la decisión de proporcionar el tratamiento a todos los niños inmediatamente después de detectado el problema. Asimismo, las autoridades de algunas escuelas deseaban ver resuelto el problema de la agresividad de los niños. Finalmente, en el presente estudio no fue posible trabajar con grupos más numerosos de niños agresivos. Tampoco fue posible estudiar muestras clínicas o de niños con cargos penales. Sin embargo, los datos aportados permiten sugerir la eficacia del tratamiento cognitivo-conductual, a los niños, y conductual, a sus padres, para disminuir la agresividad infantil. Dado que es un problema que repercute nocivamente en los ámbitos de funcionamiento de la persona agresiva, además de exacerbarse con el paso del tiempo, la intervención cognitivo-conductual representa un recurso promisorio que permitiría prevenir problemas más serios de cara al futuro. La investigación sobre la eficacia de los tratamientos aporta información importante que permite ofrecer al público la mejor atención posible.

\section{REFERENCIAS}

Achenbach, T. M. \& Rescorla, L. A. (2001). Manual for the Child Behavior Checklist. ASEBA: EEUU.

Anthony, L., Anthony, B., Glanville, D. N., Naiman, D. Q., Waanders, C. \& Shaffer, S. (2005). The relationship between parenting stress, parenting behaviour and preschoolers' social competence and behaviour problems in the classroom. Infant and Child Development, 14(2), 133-154.

Brestan, E. V. \& Eyberg, S. M. (1998). Effective psychosocial treatments of conduct disordered children and adolescents: 29 years, 82 studies, and 5, 272 kids. Journal of Clinical Child Psychology, 27, 180-189.

Caraveo-Anduaga, J. J., Colmenares-Bermúdez, E. \& Martínez-Vélez, N. A. (2002). Síntomas, percepción y demanda de atención en salud mental en niños y adolescentes de la Ciudad de México. Salud Pública de México, 44(6), 492-498.

Caspi, A. \& Moffit, T. (1995). The continuity of maladpative behavior: From description to understanding in the study of antisocial behavior. En D. Cicchetti \& D. Cohen (Eds.), Developmental Psychopatology (Vol. 2). New York: Wiley.

Castell, I., Frías, M., Corral, V. \& Sotomayor, M. (2000). Apoyo familiar, castigo corporal y sus repercusiones conductuales en niños. Revista Mexicana de Psicología, 17(1), 37-45.

Chorpita, B. F. \& Barlow, D. H. (1998). The development of anxiety: The role of control in the early environment. Psychological Bulletin, 124, 3-21.

Criss, M. M., Pettit, G. S., Bates, J. E., Dodge, K. A. \& Lapp, A. L. (2002). Family adversity, positive peer relationships, and children's externalizing behavior: A longitudinal perspective on risk and resilience. Child Development, 73, 1220-1237. 
Dumas, J. E. (1992). Conduct Disorder. In S. M. Tuner, K. S. Calhoun, \& H. E. Adams, (Eds.), Handbook of clinical behavior therapy (2nd. Ed.). (pp. 285-316). New York: Wiley.

D'Zurilla, T. J. \& Nezu, A. M. (1999). Problem solving therapy: A social competence approach to clinical intervention ( $2^{\text {nd }}$ ed.). New York: Springer.

Fergusson, D. M., Horwood, L. J. \& Ridder, E. M. (2005). Show me the child at seven: The consequences of conduct problems in childhood for psychosocial functioning in adulthood. Journal of Child Psychology and Psychiatry, 46(8), 837-849.

Forehand, R. \& Atkenson, B. M. (1977). Generality of treatment effects with parents as therapists: A review of assessment and implementation procedures. Behavior Therapy, 8, 575-593.

Froelich, J., Doepfner, M., Berner, W. \& Lehmkuhl, G. (2002). Combined cognitive behavioral treatment with parent management training in ADHD. Praxis der Kinderpsychologie und Kinderpsychiatrie, 51(6), 476-493.

Haapasalo, J. \& Pokela, E. (1999). Child rearing and child abuse antecedents of criminality. Aggression and Violent Behavior, 4 (1), 107-127.

Hamilton, K. \& Dobson, K. (2001). Empirically supported treatments in psychology: Implications for international promotion and dissemination. International Journal of Clinical and Health Psychology, 1, 35-51.

Hemphill, S. A. \& Littlefield, L. (2001). Evaluation of a short-term group therapy program for children with behavior problems and their parents. Behaviour Research and Therapy, 39 (7), 823-841.

Hernández-Guzmán, L. (1999). Hacia la salud psicológica: Niños socialmente competentes. México, D. F.: Universidad Nacional Autónoma de México.

Hernández-Guzmán, L. (2003). Lista de Conductas de Achenbach: Validez y confiabilidad. Proyecto de investigación DGAPA IN-302002 Prevención y tratamiento de la disfunción psicológica infantil. México: Universidad Nacional Autónoma de México.

Hernández-Guzmán, L. \& Benjet, C. (2003). Escala de Estrés Parental: Validez y confiabilidad. Proyecto de investigación DGAPA IN-302002 Prevención y tratamiento de la disfunción psicológica infantil. México: Universidad Nacional Autónoma de México.

Hernández-Guzmán, L., González, S. \& López, F. (2002). Effect of guided imagery on children's social performance. Behavioural and Cognitive Psychotherapy, 30, 471-483.

Huth-Bocks, A. C. \& Hughes, H. (2008). Parenting stress, parenting behavior, and children's adjustment in families experiencing intimate partner violence. Journal of Family Violence, 23(4), 243-251).

Jaffee, S. R., Caspi, A., Moffitt, T. E. \& Taylor, A. (2004). Physical maltreatment victim to antisocial child: Evidence of an environmentally mediated process. Journal of Abnormal Psychology, 113, 44-55.

Kazdin, A. E. (1987). Treatment of antisocial behavior in children: Current status and future directions. Psychological Bulletin, 102, 187-203.

Kazdin, A. E. (1996). Problem solving and parent management in treating agrressive and antisocial behavior. En E. D. Hibbs \& P. S. Jensen (Eds.), Psychosocial treatments for child and adolescent disorders: Empirically based strategies for 
clinical practice (pp. 377-407). Washington, D. C.: American Psychological Association.

Kazdin, A. E. (2001). Progression of therapy research and clinical application of treatment require better understanding of the change process. Clinical Psychology: Science and Practice. Special Issue, 8, 143-151.

Kazdin, A. \& Buela-Casal, G. (1994). Conducta antisocial. Evaluación, tratamiento y prevención en la infancia y en la adolescencia. Ediciones Pirámide: Madrid.

Kazdin, A. E., Esveldt-Dawson, K. French, N. H. \& Unis, A. S. (1987). Problem-solving skills training and relationship therapy in the treatment of antisocial child behavior. Journal of Consulting and Clinical Psychology, 55 (1), 76-85.

Kazdin, A. E., Siegel, T. C. \& Bass, D. (1992). Cognitive Problem-Solving Skills and Parent Management Training in the Treatment of Antisocial Behavior Children. Journal of Consulting and Clinical Psychology, 60 (5), 733-747.

Kingston, L. \& Prior, M. (1995). The development of patterns of stable, transient and school-age onset aggressive behavior in young children. Journal of the American Academy of Child \& Adolescent Psychiatry, 34(3), 348-358.

Landsford, J.E., Dodge, K.A., Malone, P.S., Bacchini, D., Zelli, A., Chaudhary, N., et al. (2005). Physical discipline and children's adjustment: Cultural normativeness as a moderator. Child Development, 76, 1234-1246.

Leve, L. D. \& Chamberlain, P. (2005). The development and treatment of girlhood aggression. In D. J. Pepler, K. C. Madsen, C. Webster \& K. S. Levene (Eds.), Girls in the Juvenile Justice System: Risk factors and clinical implications (pp. 191-215). Mahwah, NJ, US: Lawrence Erlbaum Associates, Publishers.

Levy, D. J. (1999). Parenting styles and the social goals of aggressive children. Dissertation Abstracts International: Section B: The Sciences and Engineering, 59 (7-B), pp. 3700.

Marshall, J. K. \& Mirenda, P. (2002). Parent-professional collaboration for positive behavior support in the home. Focus on Autism \& Other Developmental Disabilities, 17, 216-228.

Miller, B. (1989). Adult children's perception of caregiver stress and satisfaction. Journal of Applied Gerontology, 8, 275-293.

Miller, Y., Kuschel, A. \& Hahlweg, K. (2002). Early prevention of conduct disorders. Basic principles and parent-based prevention trials of externalizing behavior disorders. Praxis der Kinderpsychologie und Kinderpsychiatrie, 51 (6), 441-453.

Muris, P. , Bögels, S., Meesters C., van der Kamp, N. \& van Oosten, A. (1996). Parental rearing practices, fearfulness, and problem behaviour in clinically referred children. Personality and Individual Differences, 21(5), 813-818.

Moszcynska, U. (1979). Family environment and emergence of aggression in children at age six and seven. Psychologia Wychowawcza, 22(4), 531-539.

Nelson, D. A., Hart, C. H., Yang, C., Olsen, J. A. \& Jin, S. (2006). Aversive parenting in China: Associations with child physical; and relational aggression. Child Development, 77, 554-572.

Nietzel, M. T. \& Trull, T. J. (1988). Meta-analytic approaches to social comparisons: A method for measuring clinical significance. Behavioral Assessment, 10, 159-169.

Novak, G. (1998). A behavioral system theory of development. Mexican Journal of Behavior Analysis, 24(2), 181-196. 
Ollendick, T. H. \& King, N. J. (1998). Empirically supported treatments for children with phobic and anxiety disorders: Current status. Journal of Clinical Child and Psychology, 27, 156-167.

Patterson, G. R., Chamberlain, P. \& Reid, J. B. (1982). A comparative evaluation of a parent-training program. Behavior Therapy, 13, 638-650.

Patterson, G. R., DeBaryshe, B. D. \& Ramsey, E. (1989). A developmental perspective on antisocial behavior. American Psychology, 44, 329-335.

Peed, S., Roberts, M. \& Forehand, R. (1977). Evaluation of the effectiveness of a standardized parent training in altering the interaction of mothers and their noncompliant children. Behavior Modification, 1, 323-350.

Pinderhughes, E. E., Dodge, K. A., Bates, J. E., Pettit, G. S. \& Zelli, A. (2000). Discipline responses, influences of parents' socioeconomic status, ethnicity, beliefs about parenting, stress, and cognitive-emotional processes. Journal of Family Psychology, 14(3): 380-400.

Rhule, D. M. (2005). Take care to do no harm: Harmful interventions for youth problem behavior. Professional Psychology: Research and Practice, 36(6), 618-625.

Rubin. K. H., Burgess. K. B., Dwyer. K. M. \& Hastings. P. D. (2003). Predicting preschoolers' externalising behaviours from toddler temperament, conflict and maternal negativity. Developmental Psychology, 39(1), 164 - 176.

Sim, T. N. \& Ong, L. (2005). Parent Physical Punishment and Child Aggression in a Singapore Chinese Preschool Sample. Journal of Marriage and Family, 67(1), 85-99.

Simons, R. L., Lin, K-H. \& Gordon, L. C. (1998). Socialization in the family of origin and male dating violence: A prospective study. Journal of Marriage and the Family, 60(2), 467-478.

Smeekens, S., Riksen-Walraven, J. M. \& van Bakel, H. J. A. (2007). Multiple determinants of externalizing behavior in 5-year-olds: A longitudinal model. Journal of Abnormal Child Psychology, 35, 347-361.

Spatz-Widom, C. \& Hiller-Sturmhöfel, S. (2001). Alcohol abuse as a risk for and consequence of child abuse. Alcohol Research and Health, 25(1), 52-57.

Stormont-Spurgin, M. \& Zentall, S. S. (1995). Contributing factors in the manifestation of aggression in preschoolers with hyperactivity. Journal of Child Psychology and Psychiatry, 36(3), 491-509.

Straus, M. A., Hamby, S. L., Finkelhor, D., Moore, D. W. \& Runyan, D. (1998). Identification of child maltreatment with the parent-child Conflict Tactics Scales: Development and psychometric data for a national sample of American parents. Child Abuse and Neglect, 22, 249-270.

Su, C. W. (1975). Maternal child rearing attitudes and practices in relation to aggressive behavior of school children. Bulletin of Educational Psychology, 8, 25-44.

Tynan, W. D., Chew, C. \& Algermissen, M. (2004). Concurrent parent and child therapy groups for externalizing disorders: The rural replication. Cognitive and Behavioral Practice, 11, 99-101.

Vitaro, F., Barker, E. D., Boivin, M., Brendgen, M. \& Tremblay, E. R. (2006). Do early difficult temperament and harsh parenting differentially predict proactive and reactive aggression? Journal of Abnormal Child Psychology, 34, 685-695. 
TRATAMIENTO COGNITIVO-CONDUCTUAL DE LA CONDUCTAAGRESIVA INFANTIL 389

Wagner, B. M. (1997). Family risk factors for child and adolescent suicidal behavior. Psychological Bulletin, 121, 246-298.

Widom, C. S., Schuck, A. M. \& White, H. R. (2006). An examination of pathways from childhood victimization to violence: The role of early aggression and problematic alcohol use. Violence and Victims, 21(6), 675-690.

Webster-Stratton, C. \& Hammond, M. (1997). Treating children with early-onset conduct problems: A comparison of child and parent training interventions. Journal of Consulting and Clinical Psychology, 65(1), 93-109. 